\title{
L'entretien itinérant : pour une construction d'un dispositif méthodologique de narration des habitants dans la ville patrimoniale
}

A Methodological Tool for Narrating the Speech of Residents of a Heritage City: A Case of Mediation

Anne Watremez

\section{(2penEdition}

Journals

Édition électronique

URL : http://journals.openedition.org/edc/747

DOI : 10.4000/edc.747

ISSN : 2101-0366

Éditeur

Université Lille-3

\section{Édition imprimée}

Date de publication : 1 décembre 2008

Pagination : 77-92

ISBN : 978-2-917562-00-0

ISSN : 1270-6841

Référence électronique

Anne Watremez, «L'entretien itinérant : pour une construction d'un dispositif méthodologique de narration des habitants dans la ville patrimoniale », Études de communication [En ligne], 31 | 2008, mis en ligne le 01 décembre 2010, consulté le 20 avril 2019. URL : http://journals.openedition.org/edc/747 : DOI : 10.4000/edc.747

Ce document a été généré automatiquement le 20 avril 2019

(C) Tous droits réservés 


\section{L'entretien itinérant : pour une construction d'un dispositif méthodologique de narration des habitants dans la ville patrimoniale}

A Methodological Tool for Narrating the Speech of Residents of a Heritage City: A Case of Mediation

\section{Anne Watremez}

1 L'habiter est un fait anthropologique et constitue un "trait fondamental de l'être » (Heidegger, 1958). Il s'exprime à travers les activités pratiques dans des objets meubles et immeubles et se saisit par l'observation et par le langage, la parole de l'habitant (Segaud, 2007). Il existe un enjeu contemporain de comprendre ce que veut dire habiter une ville, et particulièrement une ville patrimoniale, terrain d'étude dans notre recherche ${ }^{1}$. Précisons que nous entendons par ville patrimoniale, ces villes dotées d'un centre historique, au patrimoine architectural et historique fortement valorisé, en cours de valorisation, ou en émergence. Nous utiliserons la même équivalence sémantique pour les expressions de ville patrimoniale et centre historique.

2 Nous souhaitons questionner ce qui se passe entre le chercheur et l'habitant de la ville patrimoniale d'Avignon à travers la parole suscitée lors d'un dispositif d'enquête. Si tout entretien sociologique a comme base un contrat de communication entre le chercheur et l'interviewé, ce dispositif de narration a de particulier qu'il se déroule en situation, in situ, c'est-à-dire dans l'espace public de la ville, qu'il allie le parler et le cheminer (c'est un entretien itinérant), et qu'il permet de faire émerger la parole de l'habitant quant à ses pratiques de l'espace urbain, et ses représentations qu'il a de sa ville. Ce dispositif méthodologique de narration permet de faire jouer pleinement à l'espace son rôle et de 
saisir « sur le vif » ses effets. Enfin, la présence physique de l'habitant dans l'espace de la ville engendre des évènements sensibles à gérer affectivement: il y a une vérité, en présence du lieu, supérieure qu'en son absence. L'entretien itinérant peut être considéré comme une forme d'interprétation de la ville patrimoniale car il constitue un dispositif qui produit une mise en discours et en regard de la ville par ses habitants.

Il s'agit par ailleurs de montrer que l'habitant devient un acteur producteur de la ville, un acteur sociosémiotique. Il est ainsi à la fois et en même temps un pratiquant de la ville qui s'approprie l'espace; un usager qui se distingue selon les pratiques et les fréquentations qu'il a de l'espace et un citoyen qui se pose en responsable de la question humaine. Ainsi nous interrogeons l'ensemble des relations qui s'établissent entre le chercheur et l'habitant dans une attitude réflexive: qu'est-ce qui se joue dans la médiation du dispositif méthodologique effectué dans la ville patrimoniale? Quels types de relations, quels rôles se mettent en place dans l'économie de l'entretien itinérant? En quoi l'ensemble de cette réflexivité permet de comprendre ce que veut dire habiter une ville?

Il est nécessaire avant tout de procéder à une brève description du terrain d'étude, dont la configuration spatiale et patrimoniale constitue une identité forte en soi.

\section{Les caractéristiques d'une ville patrimoniale}

5 La ville patrimoniale est dotée d'un centre historique considéré comme un secteur présentant un caractère historique, esthétique ou de nature à justifier la conservation, la restauration et la mise en valeur. Son tissu urbain est hérité des villes médiévales, il est constitué de rues étroites, d'une certaine hauteur des bâtiments, et de l'omniprésence du patrimoine. Les institutions de ces villes donnent à voir ce patrimoine, le médiatisent à travers de nombreux dispositifs, dans le cadre d'une politique touristique forte à travers des labels (Ville et Pays d'Art et d'Histoire, Patrimoine mondial de l'humanité). Elles voient ainsi passer dans leurs murs des milliers, voire des millions de touristes, et, parfois, la cohabitation avec les habitants ne se fait pas en toute sérénité.

6 Le centre historique de la ville patrimoniale est par ailleurs un vecteur de l'identité urbaine. Cette valeur symbolique accordée au centre urbain n'est apparue qu'après de nombreuses années de débat au milieu des années $60^{2}$. Une approche urbaine globale se développe, considérant que l'intérêt historique, culturel et esthétique de nombreuses villes ne pouvait être réduit à la seule présence d'éléments remarquables, mais qu'il résidait aussi dans l'harmonie et la qualité de l'ensemble des édifices et des espaces qui le composent. La loi du 4 Août 1962 a consacré juridiquement cette extension du champ patrimonial aux ensembles bâtis en créant les secteurs sauvegardés.

7 Avignon est une de ces villes patrimoniales qui, grâce à son patrimoine et son festival international de théâtre, voit entrer dans ses remparts quelques deux millions de touristes chaque année. Elle a une personnalité spatiale forte: construite sur un rocher (le rocher des Doms) au pied duquel se trouve un fleuve (le Rhône), elle est une ville remparts, le centre-ville historique est situé dans un intra muros - héritage du tissu urbain médiéval européen -, opposé à un extra muros qui regroupe les autres quartiers principalement construits dans la deuxième moitié du $\mathrm{xx}^{\mathrm{e}}$ siècle. L'intra muros est un périmètre urbain protégé par la loi, une partie de son patrimoine est inscrite au patrimoine mondial depuis $1995^{3}$, il est ce que nous appelons ici le centre historique. Il possède un patrimoine monumental exceptionnel, caractérisé par une richesse 
architecturale de monuments construits du XIV ${ }^{\mathrm{e}}$ au XVIII ${ }^{\mathrm{e}}$ siècles, hérité de la présence de la papauté (construction de palais, couvents, églises, livrées, hôpitaux, hôtels particuliers). Les institutions municipales ont développé une politique culturelle autour d'une «image de marque patrimoniale» axée sur deux «monuments»: le Palais des Papes/Pont d'Avignon inscrit au patrimoine mondial et le Festival international de Théâtre crée par Jean Vilar. Ce caractère patrimonial prégnant a des conséquences dans les différentes pratiques de la ville parce que le patrimoine fait partie intégrante de son espace, de son identité urbaine.

\section{Les notions d'habitant et d'habiter ou la question de la pratique ordinaire de la ville}

8 Nous souhaitons dans un premier temps discuter épistémolo-giquement la notion d'habitant. Suffit-il de dire qu'elle s'oppose à celle du touriste ? Il s'agit de clarifier ici ces notions utilisées dans des disciplines aussi diverses que la géographie, la philosophie, la sociologie.

\section{Les sens de l'habiter ${ }^{4}$}

9 L'habiter ${ }^{5}$ 'est, dans un espace et un temps donnés, tracer un rapport au territoire en lui attribuant des qualités qui permettent à chacun de s'y identifier.

10 En géographie et sociologie urbaine, habiter est défini comme avoir son domicile en un lieu, occuper un logement, résider. Les habitants sont alors des résidents avec le statut juridique qui confère un droit.

11 C'est la philosophie phénoménologique qui donne à la notion de l'habiter sa dimension ontologique et anthropologique. Heidegger dans le chapitre "Bâtir Habiter Penser » (1958) pose les bases d'une conception de l'habiter pour en faire une activité primordiale, constitutive de l'être humain. Il établit une séparation radicale entre l'habiter et sa mise en rapport poétique avec le monde et le fait de se loger, en tant que simple acte fonctionnel. Comme la pensée humaine précèderait sa matérialité, l'habiter précèderait le bâtir. Selon la phénoménologie, habiter c'est comprendre le sens du rapport entre l'individu et son environnement, c'est penser l'individu comme acteur d'une partie de sa réalité géographique (Lazzarotti, 2006).

12 Bachelard, dans La poétique de l'espace (1958), montre que l'espace habité se comprend aussi par les sensations corporelles. Cet habiter ne se cantonne plus à l'étude de la maison et de l'intime, il se situe au niveau du corps et est associé au quotidien. L'espace habité est celui qui est investi émotionnellement. La psychologie sociale de Fischer (1981) vise alors l'étude des relations qui s'établissent entre les hommes et les espaces. Cet auteur est à l'origine, avec Hall (1971), d'un néologisme, celui de proxémie définie comme l'ensemble des observations et théories concernant l'usage que l'homme fait de l'espace en tant que produit culturel spécifique.

13 Avec Lefebvre dans les années 70 , l'habiter s'avère être une compétence socialement acquise et insérée dans les habitus. La dimension sociale de l'habiter et non sa fonction est privilégiée. Cette conception place au centre du projet urbain le social et s'inscrit dans le cadre problématique de la ville. 

des hommes, aux nouvelles formes de mobilités géographiques et au tourisme. Pour cette équipe, la notion de déplacement est le point de départ de l'interrogation sur l'habiter. Cette réflexion est inspirée par l'évolution des pratiques de mobilités. Le phénomène du tourisme est défini ici comme un déplacement, un changement de place, un changement d'habiter: le touriste quitte temporairement son lieu de vie et habite temporairement dans d'autres lieux. Ainsi, la dimension géographique participe pleinement à la constitution de l'être humain. Lazzarotti (2006) développe l'idée qu'en habitant les lieux et les territoires, en y résidant, en les fréquentant, en les traversant, les hommes participent individuellement et collectivement à la construction du monde.

Stock définit l'habiter comme le fait d'être à la fois physiquement dans un lieu mais aussi de « rapporter » des pratiques de lieux extérieurs, dans son lieu de vie. On peut donc avoir une approche de l'habiter par l'ensemble des pratiques qu'un individu associe à des lieux. Pratiquer les lieux, c'est en faire l'expérience et déployer en actes un faire qui a une certaine signification pour les hommes. La notion d'habiter ici prend une nouvelle dimension : il s'agit de l'articulation entre pratiques et significations des lieux. Nous nous inscrivons dans cette dernière posture.

\section{L'habiter comme ensemble de pratiques sensibles des lieux}

16 La recherche a permis de construire la notion d'habitant par le médium de la pratique des lieux de la ville (ou pratique citadine), faite à la fois de trajets et d'ambiances. Elle est ce que les individus font avec les lieux et ces manières de pratiquer ont du sens. La pratique citadine peut être ordinaire ou non, relevant du normal, du banal, de l'habituel, dans l'activité de la ville. Elle peut être quotidienne en ce sens où l'activité se répète tous les jours, ou pas.

17 La pratique citadine, passée comme actuelle, est ainsi le terreau à partir duquel les parcours, lors de l'entretien itinérant sont construits. On peut ainsi en repérer cinq types dont la pratique de la ville décrite se situe dans le passé, et/ou dans le présent: un parcours reprend les trajets effectués pendant l'enfance, on passe alors devant les anciens lieux de vie ; un autre constitue celui de la vie ordinaire ; un parcours revisite les lieux « d'avant » c'est-à-dire les anciens lieux d'habitation ou de savoirs ; certains symbolisent l'identité familiale, plus précisément la construction de l'identité par l'impact de la vie de famille dans l'espace urbain, le parcours prend alors l'apparence d'un pèlerinage ; enfin, un parcours constitue une redécouverte de la ville pour l'habitant.

18 Mais la pratique citadine n'est pas uniquement constituée de ces trajets, elle est un espace des sensibles qui fait appel à nos cinq sens. L'espace formel de la ville, la présence du soleil, des odeurs, de la végétation sont essentiels dans l'appréhension de cette pratique et constitue l'ambiance d'une ville. Celle-ci a été théorisée par le laboratoire Cresson à Grenoble: elle est l'expérience sensible de la ville et passe par un ensemble de phénomènes localisés pour exister ${ }^{7}$ : la perception du citadin, la mise en forme de la ville (formes architecturales, matériaux utilisés...), son contexte environnemental (sons, lumières...). Avignon est ainsi la "ville du vent violent $»^{8}, \mathrm{du}$ mistral, qui, quand il s'engouffre dans les rues étroites, déforme le corps et la marche. Certaines rues sont recouvertes de "calades", ces galets ${ }^{9}$ qui modifient le pas. Elle est une ville lumineuse dont les couleurs dominantes sont celle de la pierre, omniprésente, et du bleu du ciel par temps de mistral, elle est la ville aux clochers et cloches qui ponctuent le temps urbain.

Études de communication, 31 | 2008 
Ville mystérieuse, elle s'inscrit dans un hors temps, dans une médiévalité et italianité fortes: petites ruelles étroites, omniprésence de la pierre, bâti serré, calades ${ }^{10} \ldots$ L'ambiance de la ville d'Avignon relève d'une certaine authenticité et se rapproche de ce que Riegl appelle la valeur d'ancienneté, qui constitue l'ensemble des traces d'ancienneté, des effets subjectifs et affectifs liés à un patrimoine. Elle engendre un engagement du sujet dans la subjectivité, la sensibilité et l'affectivité, s'adressant à tous les individus sans distinction de niveau culturel.

Envisager l'importance de l'expérience sensible de l'espace urbain, considérer qu'il y a un engagement du sujet percevant le milieu social et les qualités physiques et sensibles de l'environnement, qu'il y a mis en jeu des sens, du corps, de la motricité du citadin permettent la mise en place du dispositif méthodologique de narration, l'entretien itinérant.

\section{La médiation de l'entretien itinérant : entre expertise et partage d'émotions}

Nous travaillons ici sur une partie du corpus constitué d'entretiens itinérants effectués de mars à octobre 2007 auprès de treize habitants ${ }^{11}$. Leur durée varie de une et à deux heures trente. Il constitue ce que Derèze (1997) appelle les discours provoqués c'est-à-dire stimulés, encouragés, imposés par le chercheur incitant les acteurs sociaux à "faire part » ou à raconter. Cette classification des discours se fait non en fonction de celui qui parle ou la connaissance des faits mais en fonction de la qualité de la relation sociale instaurée par les discours entre l'habitant-acteur et le chercheur.

L'échantillon s'est construit selon le principe de l'effet «boule de neige », en puisant à chaque rencontre dans le réseau de sociabilité de chaque interviewé : une première sphère est constituée de personnes que nous connaissions dans le cadre de notre pratique citadine ; une seconde sphère constituée de personnes inconnues dont nous avons eu les noms par la première; une troisième sphère construite sur le même principe que la précédente et ainsi de suite. La rencontre préalable à l'entretien itinérant est indispensable pour valider le contact selon la définition de l'échantillon ${ }^{12}$, évaluer la motivation et établir le contrat de communication: donner la consigne, la durée approximative de l'itinérance, parler de l'autorisation de l'enregistrement de l'entretien, de la reproduction du tracé du parcours réalisé sur un plan. Un rendez vous est pris pour la réalisation de l'entretien itinérant ${ }^{13}$.

\section{La valeur heuristique du dispositif méthodologique de narration : pour une mise en discours de la ville par l'habitant}

L'entretien itinérant, co-construit par le chercheur en interaction avec l'habitant, constitue une mise en discours et en regard de la ville patrimoniale en faisant prendre conscience à l'habitant de pratiques impensées. Le dispositif de narration permet donc de faire énoncer un ensemble de pratiques et de représentations au caractère impalpable et ténu. Il y a par ailleurs une autorité partagée entre enquêteur et enquêté, constituant un mode de production de savoir c'est-à-dire « une négociation construite impliquant au minimum deux sujets conscients, politiquement significatifs » (Clifford, $1988: 48$ ). 

deux dimensions ont été traitées dans différentes disciplines et procèdent d'enjeux méthodologiques importants. Pour le Marcher, il s'agit de prendre en considération l'existence d'une tradition de l'analyse des parcours dans l'espace dans des disciplines telles que la sémiotique, la muséologie et la sociologie urbaine; et pour le Parler, de la tradition de l'entretien compréhensif ${ }^{14}$. Le parcours est construit par l'interviewé, il peut être réfléchi si l'intervalle de temps entre le $1^{\text {er }}$ entretien de présentation de la consigne et le second qui est l'entretien itinérant en soi est utilisé à cet effet (certains ont même une liste de «lieux» inscrits sur une feuille), ou spontané, improvisé. Concernant la consigne, nous commençons généralement par demander aux gens de nous emmener sur les lieux qu'ils pratiquent ou ont pratiqué, qu'ils apprécient particulièrement, qui leur évoquent un souvenir lié à une expérience, les lieux qu'ils font visiter à leurs amis quand on leur rend visite. On leur demande de refaire leurs trajets quotidiens (du domicile au lieu de travail par exemple).

Quels sont donc les enjeux de l'entretien itinérant, de son énonciation? En quoi permet-i la production, l'expression de mémoires, d'imaginaires, d'interprétations des habitants?

Au-delà d'une "vérité » certaine d'être devant le lieu, l'entretien itinérant permet de reconstituer les conditions physiques de la pratique ordinaire : bruits de la ville, voitures, foule, mistral, désorientation : tout ceci concourt à mettre en situation réelle l'habitant. Les lieux, par ailleurs, interpellent, interfèrent lors de l'itinérance : il y a ainsi le parcours pensé par l'habitant et celui spontané qui s'improvise face à la vue d'un édifice.

L'entretien itinérant a la faculté de révéler la supériorité de la dimension sensible des signes dans les processus de signification et les modalités de compréhension de l'environnement urbain. Pour cela, le non assertif doit être repéré : les silences - plus ou moins longs, plus ou moins gênants pour l'enquêté - marquent le recueillement, la réflexion, l'écoute, la tristesse. Les intonations de voix sont relevées - on chuchote par respect devant un lieu en particulier (par exemple devant l'institution culturelle cinématographique d'Avignon pour un cinéphile). L'utilisation du corps est observée : on éprouve le besoin de toucher les murs de pierre pour se remémorer, pour s'imprégner d'un lieu ou pour accentuer un propos. La mise en récit de la ville patrimoniale par l'habitant permet même de décrire des traces sensibles urbaines disparues :

il y avait une usine Laugier [...] sans arrêt il y avait ces machines qui faisaient un grand bruit, un bruit sourd et je me souviens dans la chambre, dans cette maison là, la chambre qui est le plus de ce côté, mais il y avait un espèce de bourdonnement sourd et je me souviens que je l'entendais [...] c'était même réconfortant [...], ça faisait une

espèce de respiration de la ville et de la même façon qu'on entendait de là les sirènes des bateaux, des péniches sur le Rhône [...] tout ça ça faisait les rumeurs «ce que dit cette paisible rumeur là vient de la ville », et ça faisait des rumeurs que moi en tant que gamin je trouvais réconfortantes par rapport à mes parents qui habitaient en pleine campagne, là on est dans la ville et ça faisait quelque chose de chaud, d'humain, une compagnie qui était moi je me souviens agréable // et puis alors ces sirènes de brumes c'était d'une poésie folle, c'était fabuleux, c'était du Stevenson! [Entretien itinérant $\mathrm{n}^{\circ} 8$ ].

Une des caractéristiques de l'entretien itinérant est la forte présence de la gestuelle pour appuyer un propos ou désigner les lieux. Les déictiques « ça », «là », « ici » sont utilisés plutôt que la nomination des lieux. Les embrayeurs ${ }^{15}$ spatiaux sont en plus grand nombre que les embrayeurs temporels. La proximité affective à un lieu, marquée par l'utilisation 
des pronoms possessifs « ma ville », « ma rue », «mon quartier », peut s'exprimer pour et par l'habitant seul ou au nom de la collectivité, c'est alors des terminologies comme «notre prison », «notre épicier» qui sont utilisées pour accentuer la présence d'une mémoire sociale d'un quartier en danger ou disparue.

L'entretien itinérant renseigne sur les manières de pratiquer la ville : elles oscillent de la déambulation avec comme objectif la désorientation et la découverte de nouveaux lieux à l'association de sa manière de pratiquer la ville avec les pratiques culturelles des enquêtés dans l'espace urbain: photographie, dessin, festival de théâtre (il existe des rues pratiquées uniquement pendant le festival), cinémas, ou encore en fonction d'une passion: une habitante a construit une pratique de la ville en fonction des lieux de tournage ayant eu lieu dans Avignon et sa région. Cette femme, se qualifiant de très cinéphile, ayant fait tous les castings en tant que figurante dans ces films, avoue avoir fait du théâtre et son rêve non réalisé de « faire des films ».

Enfin, le style général ${ }^{16}$ de l'entretien itinérant donne des informations sur les marques d'énonciation qui manifestent un point de vue, une opinion, un dispositif d'argumentation. Les éléments pris en compte pour définir ce style général du texte sont la mise en scène verbale - verbes d'actions ou verbes d'état - la prise en charge de l'énoncé, et le style du texte - argumentatif, narratif, énonciatif ou descriptif.

Si tous les entretiens itinérants ont une mise en scène ancrée dans le réel (présence principalement de verbes d'état) et pris en charge à l'aide du «je », les styles du texte sont de deux ordres.

31 D'une part, le style argumentatif, majoritaire, où le sujet s'engage, argumente, explique ou critique pour essayer de persuader l'interlocuteur. Le parcours effectué lors de l'entretien itinérant est, dans ce cas, tourné vers des lieux associés au passé (refaire des trajets effectués pendant l'enfance, parcours des lieux "d'autrefois») et au présent (parcours de la vie ordinaire, de l'identité familiale, de redécouverte de la ville). D'autre part, le style énonciatif, minoritaire, où le locuteur et l'interlocuteur établissent un rapport d'influence et révèlent leurs points de vue. Le parcours est alors uniquement tourné vers des lieux évoquant le passé. Dans ces entretiens, les habitants qui parlent sont nés à Avignon et/ou ont une pratique ancienne de la ville de plus de vingt ans. Ce n'est pas tant la durée de cette pratique qui fait le style du texte (on retrouve ce profil dans le style argumentatif) mais plutôt l'investissement émotionnel dont ils font preuve, de la vivacité de leurs souvenirs encore extrêmement précis liés à leur pratique de la ville pendant l'enfance, tranche de vie où l'ancrage de la famille dans l'espace urbain s'est inscrit. Ainsi on se souvient, outre des lieux de vie classiques comme l'école, le jardin, le collège etc... du magasin des premiers achats de disques, de la boulangerie, du petit lait, de l'appartement du médecin dont on avait « une trouille terrible », de passages dérobés, certains aujourd'hui disparus. C'est surtout la palette des émotions ressenties devant ces lieux du temps de l'enfance qui refont surface et sont clairement décrites. Ainsi tout un ensemble de traces et d'indices de la vie passée permet de déployer le récit familial.

\section{Un ensemble de relations entre habitant et chercheur : de l'échange d'expertise...}

Un premier type de relations est l'échange relevant de l'expertise. Certains habitants se positionnent comme érudits, ils sont possesseurs d'un savoir qu'eux seuls semblent 
connaître : des connaissances sur des lieux, sur leur quartier, des anecdotes, des « petites choses » découvertes lors de la pratique de la ville, comme les signatures des ouvriers situées sur les pierres d'un jardin constituant un mur. Inversement, l'habitant peut considérer le chercheur comme un expert de la ville: il est donc sollicité pour qu'il partage ses connaissances de/sur la ville, sur des questions de connaissances (en histoire de l'art par exemple), sur sa manière de pratiquer la ville. L'interviewer peut aussi être considéré comme un "porte-parole ", un représentant des institutions municipales, un relais d'informations.

Ces échanges d'expertise se font principalement sur des questions de politiques culturelles et urbaines de la ville. Les registres civique et de l'indignation sont convoqués : certains habitants, adhérents à des associations de sauvegarde et de défense de quartiers, suivent le moindre projet de réaménagement, de réhabilitation, de restauration. L'entretien devient une tribune pour contester les projets urbains constituant, selon eux, une "homogénéisation", une "standardisation", une "perte d'âme et d'identité ». Ainsi, face à la perte engendrée par le projet de réhabilitation d'une place, de ce qu'une habitante appelle « le construit spontané qui se fait par la pratique des lieux, constituant des couches successives de l'histoire du lieu », celle-ci est abandonnée et disparaît de la pratique de la ville. L'habitante fait part de sa crainte du phénomène de muséification et de gentrification ${ }^{17}$ de la ville : elle doit conserver avant tout une diversité dans sa vie sociale.

L'habitant peut jouer le rôle d'un guide complice auprès du chercheur. Il lui révèle les petites histoires et non la grande Histoire de la ville comme celle de se rendre en cachette dans les réserves d'un musée pour s'y « servir » ou la révélation d'un passage secret pour se rendre au Palais des Papes caché derrière une cheminée dans le hall de la maison familiale.

\section{...aux partages d'émotions}

Nous l'avons dit, pratiquer les lieux c'est en faire l'expérience, c'est leur donner un sens et leur accorder une dimension symbolique qui change en fonction des habitants, permanents ou temporaires. La dimension sensible des lieux, leurs expériences et leurs capacités à évoquer des souvenirs sont alors des modalités de construction du sens des lieux. Nous avons la conviction que ces dimensions priment lors de la mise en œuvre du dispositif, le refus d'un de nos contacts de faire l'exercice parce que «ce serait trop douloureux » étant un indice.

Ainsi, pour certains, la pratique de la ville est liée à une construction ontologique de la personne où l'expérience de la famille à partir de différents lieux devient centrale. Lors de l'entretien itinérant, l'habitant retourne dans le quartier et les lieux fréquentés pendant l'enfance. Le lieu symbolise alors le bonheur ou la douleur de ces années. Des moments de vie vécus autrefois resurgissent : faire du patin à roulette et jouer au foot dans les halls des maisons familiales, achat des deux premiers disques dans un magasin disparu, les inondations du Rhône...

37 Certains mènent une réflexion sur ce bonheur retrouvé : un enseignant de lettres, né à Avignon, n'était pas revenu dans le quartier où se trouve la maison de ses grands parents depuis fort longtemps, il analyse l'émotion qu'il ressent pendant l'entretien et fait référence au bonheur proustien. Face aux maisons décaties de son ancien quartier, le mécanisme de la madeleine de Proust est activée. Pour expliquer l'émotion, la joie de ce 
temps retrouvé, cet habitant s'identifie à Proust qui connaît lui-même, en tant que narrateur, ce phénomène décrit dans différents passages de son œuvre. L'interviewé nous fait partager son amour de Proust, jusqu'à réciter les passages évoqués :

Par exemple moi ce que je ressens ici, c'est quelque chose de très précis et de très net [...], moi ça [la maison de ses grands parents] ça me fait cet effet, c'était décati à l'époque comme c'est décati maintenant, ça a pas bougé, mais vraiment au millimètre, la peinture c'était un peu plus lisible à l'époque, ça n'a pas bougé [...]. Oui c'est vieux ça, les volets qui s'articulent et qui se cachent dans l'embrasure ça n'a pas bougé / alors l'analyse que fait Proust c'est que le temps est vaincu c'est-àdire qu'il est là le bonheur, c'est un bonheur d'avoir vaincu le temps, c'est-à-dire que je suis là et je vois ça exactement, ce qui fait qu'au travers des années l'individu reste le même, le temps s'est écoulé mais l'individu a vaincu le temps, c'est ça le fond de la joie, du bonheur proustien de ses souvenirs [entretien itinérant $n^{\circ} 8$ ].

Et en effet, Proust, à travers les épisodes de la madeleine et des pavés dans la cour de l'hôtel de Guermantes, souhaite évoquer le phénomène de la mémoire involontaire surgissant avec l'odeur et la saveur. Cette mémoire donne lieu à une analogie entre une de ces sensations du passé et une sensation fortuite du présent. Une vie s'écoule sans que l'individu en ait conscience et seul un évènement fortuit fait surgir à la conscience le passé dans son ensemble et comprendre que seul le temps écoulé, « perdu », a une valeur.

\section{Conclusion}

L'article a voulu questionner la notion d'habitant dans un contexte particulier, la ville patrimoniale, à travers la mise en place d'un dispositif de communication, l'entretien itinérant. La notion d'habitant a ainsi été construite autour de la pratique de la ville et de la prise en compte de l'expérience sensible de l'espace urbain.

Nous avons focalisé notre réflexion sur l'énonciation du dispositif construit pour la recherche, énonciation dont nous avons essayée de montrer la pertinence avant d'en repérer ses enjeux et l'ensemble des relations entre le chercheur et l'habitant lors de sa mise en œuvre. Les pistes de résultats, parcellaires dans le cadre de cet article, doivent être poursuivies afin de montrer toute la richesse de l'économie de l'entretien itinérant.

Il s'agissait par ailleurs de développer, non pas les représentations et interprétations des habitants en soi mais les moyens de mise en parole et en discours de la ville par les habitants. C'est un questionnement qu'il nous faut poursuivre, à savoir comment l'habitant construit son identité spatiale, sa relation à la ville, notamment par sa pratique des lieux et non uniquement en passant par l'étude des représentations.

\section{BIBLIOGRAPHIE}

Augoyard, J.-F., (1979), Pas à pas : Essai de cheminement quotidien en milieu urbain, Paris, Éd. du Seuil. 
Bachelard, G., (1998-1964), La Poétique de l'espace, Paris, Presses Universitaires de France [1 ère édition 1957].

Clifford, J., (1988), « De l'autorité en ethnographie », pp. 29-59, in : Malaise dans la culture. L'ethnographie, la littérature et l'art du XXe siècle, Paris, Éd. École nationale des beaux arts [traduit de l'américain par Marie Anne Sichère].

Da-Lage, É. \& Gellereau, M., (2006), « L'expert et l'amateur : valoriser l'interprétation du patrimoine urbain par les habitants ", communication au colloque international Les Arts de la Ville dans la prospective urbaine, Université de Tours, 9-10 mars 2006.

De Certeau, M., (1990), L'Invention du quotidien, t. 1, « Arts de faire », Paris, Gallimard.

Derèze, G., (1997), « De la 'vie sociale' des récits médiatiques. Une ethnosociologie focalisée », pp. 121-134, in : Recherches en communication, $\mathrm{n}^{\circ} 7$.

Fischer, G., (1981-1964), La Psychosociologie de l'espace, Paris, Presses Universitaires de France (QSJ).

Hall, E. T., (1971-1966), La Dimension cachée, Paris, Le seuil (Essai/points).

Heidegger, M., (1958-1951), « Bâtir habiter penser », Essais et Conférences, Traduit de l'Allemand par André Préau, Paris, Gallimard [« Bauen Wohnen Denken », in : Vorträge und Aufsätze], pp. 170-193.

Lazzarotti, O., (2006), Habiter, la condition géographique, Paris, Belin (Mappemonde).

Maingueneau, D., (1998), Analyser les textes de communication, Paris, Nathan (Lettres sup.).

Segaud, M., (2007), Anthropologie de l'espace. Habiter, fonder, distribuer, transformer, Paris, Armand Colin (U. Sociologie).

Stock, M., (2006), « Construire l'identité par la pratique des lieux », Chez Nous. Territoires et identités dans les mondes contemporains, sous la direction de Alessia De Biase et Christina Rossi, Paris, Éd. la Villette, pp. 142-159.

Stock, M., (2004), «L'habiter comme pratique des lieux géographiques », <www.espacestemps.net/document1138.html> (Consulté le 12 avril 2007).

Watremez, A., (2008), « Vivre le patrimoine urbain au quotidien : pour une approche de la patrimonialité », pp. 11-35, Culture et Musées, $n^{\circ} 11$.

\section{NOTES}

1. Cette recherche de doctorat a pour titre La Patrimonialité: pour une approche de la construction du regard patrimonial des habitants à travers la pratique ordinaire de la ville. Cette thèse en cours se fait au sein du laboratoire Culture et Communication, de l'université d'Avignon et des Pays de Vaucluse.

2. Les raisons de cette non reconnaissance sociale et symbolique du centre urbain sont de trois ordres : son échelle et sa complexité, l'absence de cadastres et de documents cartographiques fiables, la persistance de la mentalité identifiant la ville à un nom, à une communauté, à une généalogie, à une histoire personnelle mais qui se désintéresse de son espace urbain.

3. L'ensemble monumental pontifical inscrit au patrimoine mondial de l'humanité est constitué de l'ensemble de monuments suivants: le Palais des Papes et sa place, le Pont saint Bénézet, le cathédrale Notre Dame des doms, le Petit Palais, les tours des Chiens et du Châtelet des remparts et le morceau de rempart entre ces deux tours. 
4. Pour reprendre le titre d'un chapitre d'Olivier Lazzorotti «Du faible au fort: les sens de l'habiter", in : Habiter, la condition géographique, 2006, Paris : Belin. Ce chapitre constitue une très bonne synthèse des conceptions de l'habiter dans les différentes disciplines en sciences sociales.

5. L'Habiter utilisé en tant qu'infinitif substantivé constitue le phénomène anthropologique général.

6. Mobilités, Itinéraires, Territoires, équipe de recherche à Paris 7 - Denis Diderot.

7. Pour une définition plus détaillée, voir Augoyard, «Éléments pour une théories des ambiances architecturales et urbaines ", in : Les cahiers de la recherche architecturale, 1977, pp. 13-23.

8. «Aouen(n)ion", un nom d'origine cavare (le peule celto-ligure qui habite le rocher 500 ans av. J.-C.), a deux interprétations : «ville du vent violent » ou encore «seigneur du fleuve » selon que la traduction est faite à partir du celte ou du ligure. Il semble néanmoins que la seconde interprétation soit plus vraisemblable.

9. Les galets viennent du Rhône, les calades qui datent de l'époque médiévale ont été restaurées, elles ne sont pas d'époque.

10. Ses différents résultats sont issus d'une série de mini questions pour connaître l'image de la ville : notamment à la question «Si Avignon était une couleur ", la pierre et le ciel bleu du mistral arrivent en majorité, à la question "Si Avignon était un livre», Les villes invisibles d'Italo Calvino (car la ville est bien présente mais garde encore sa part de mystère et d'impalpable) et La chambre des Dames de Jeanne Bourrin et Le nom de la rose d'Umberto Eco (pour le cadre médiéval de la ville) sont mentionnés.

11. Deux couples ont choisi de faire l'entretien itinérant ensemble. Il a alors été demandé que chacun prenne son tour de parole.

12. Ces variations sont le lieu de résidence et de travail, le mode de déplacement, l'ancienneté de l'installation, le lieu de naissance, le type d'habitation et le talon sociologique classique.

13. Pour plus de détails sur la construction du dispositif de l'entretien itinérant en lien avec la question de recherche, voir Watremez A. (2008). «Vivre le patrimoine urbain au quotidien : pour une approche de la patrimonialité », pp. 11-35, Culture et Musées, $n^{\circ} 11$.

14. Idem.

15. Selon Maingueneau (1998), on appelle embrayage l'ensemble des opérations par lesquelles un énoncé s'ancre dans sa situation d'énonciation et embrayeurs ou déictiques les éléments qui dans l'énoncé marque cet embrayage. Il existe des embrayeurs de personne (pronoms personnels, déterminants ou pronoms possessifs), des embrayeurs temporels et spatiaux : ces derniers se distribuent à partir du point de repère que constitue le lieu où se tient l'énonciation. "Ici » désigne la zone même où parlent les coénonciateurs, « là-bas » un endroit éloigné, «ça » un objet inanimé montré. On trouve aussi des groupes nominaux déterminés par «ce». L'embrayeur permet au co-énonciateur d'identifier le référent.

16. Cette identification du style général du texte est effectuée grâce au logiciel Trope.

17. Muséification signifie que le centre historique devient un musée. Gentrification de gentry, (petite noblesse en anglais), ou " embourgeoisement », en tant que processus par lequel le profil économique et social des habitants d'un quartier se transforme au profit d'une couche sociale supérieure; on ne retrouve plus qu'une seule classe sociale dans le même périmètre urbain, le centre historique. 


\section{RÉSUMÉS}

Nous souhaitons comprendre ce que veut dire aujourd'hui habiter une ville patrimoniale en questionnant d'une part ce qui se passe entre le chercheur et l'habitant de la ville patrimoniale d'Avignon à travers la parole suscitée lors d'un dispositif d'enquête (l'entretien itinérant) ; et d'autre part montrer que l'habitant est ainsi à la fois et en même temps un pratiquant ordinaire de la ville qui s'approprie l'espace; un usager qui se distingue selon les pratiques et les fréquentations qu'il a de l'espace et un citoyen qui se pose en responsable de la question humaine.

Nous interrogeons l'ensemble des relations qui s'établissent entre le chercheur et l'habitant dans une attitude réflexive: qu'est-ce qui se joue dans la médiation du dispositif méthodologique effectué dans la ville patrimoniale ? Quels types de relations, quels rôles se mettent en place dans l'économie de l'entretien itinérant? En quoi l'ensemble de cette réflexivité permet de comprendre ce que veut dire habiter une ville?

La première partie s'attache à discuter épistémologiquement la notion d'habitant. Il s'agit de clarifier ici ces notions utilisées dans des disciplines aussi diverses que la géographie, la philosophie, la sociologie. La seconde partie explique comment le chercheur a fait émerger la parole de l'habitant sur sa pratique de la ville au moyen d'un dispositif méthodologique de narration : l'entretien itinérant. La mise en œuvre de celui-ci, co-construction du chercheur et de l'habitant, permet de repérer l'ensemble de relations qu'il est intéressant de mettre à jour. Elles vont de l'expertise au partage d'émotions.

This paper considers what it means today to live in a heritage city not only by investigating what takes place between the researcher and residents of the heritage city of Avignon through the discourse generated by a survey consisting of itinerant interviews, but also by showing that residents are also ordinary practitioners of the city who appropriate space, users who are distinguished according to the practices and uses they exhibit in that space, and citizens positioned as being in charge of the human question. The paper questions all of the relations created between the researcher and residents in a reflexive manner. What plays out in the mediation of the methodological process conducted in a heritage city? What types of relations and roles are set up in the enunciation of itinerant interviews? In what sense does this reflexivity make it possible to include what it is to live a city?

The first part of the paper discusses the concept of resident. The aim is to clarify these concepts as used in disciplines as various as geography, philosophy, and sociology. The second part explains how the researcher allows residents' experience to emerge in their utterances on urban practices through the methodological device of narration as used in itinerant interviews. The implementation of such interviews, which implies co-construction by researcher and resident, makes it possible to locate all of the whole of relations that can be revealed and that range from expertise to emotions. 
INDEX

Keywords : inhabitant, practice, city, heritage city, speech, researcher, space, discussion

Mots-clés : habitant, pratique, ville, discours, ville patrimoniale, chercheur, espace, entretien

\section{AUTEUR}

\section{ANNE WATREMEZ}

Anne Watremez est doctorante dans le programme international conjoint en muséologie, médiation et patrimoine de l'Université du Québec à Montréal et l'Université d'Avignon et des Pays de Vaucluse. Sa thèse, intitulée La patrimonialité : pour une étude des représentations patrimoniales ordinaires dans la ville, se fait sous la direction de Luc Noppen (UQAM) et de Jean Davallon (UAPV). Elle est actuellement ATER au sein du département Sciences de la Communication de l'Université d'Avignon et des Pays de Vaucluse. 